\title{
Re: Prolonged Warm Ischemia Time is Associated With Graft Failure and Mortality After Kidney Transplantation
}

\author{
Tennankore KK1, Kim SJ2, Alwayn IP3, Kiberd BA1 \\ 1Dalhousie University Faculty of Medicine, Department of Nephrology, Nova Scotia, Canada; Nova Scotia Health Authority, Nova Scotia, Canada \\ 2University Health Network, University of Toronto Faculty of Medicine, Department of Nephrology, Ontario, Canada; Institute of Health Policy, University of \\ Toronto Faculty of Medicine, Department Management and Evaluation, Ontario, Canada \\ 3Dalhousie University Faculty of Medicine, Department of Surgery, Multi-Organ Transplant Program, Nova Scotia, Canada
}

Kidney lnt 2016;89:648-658. doi: 10.1016/j.kint.2015.09.002. Epub 2015 Dec 30.

\section{EDITORIAL COMMENT}

The effect of warm ischemia time (the time from organ removal from cold storage to reperfusion with warm blood) on death and graft survival was investigated on a very large cohort of kidney recipients consisting of 131677 patients with 35901 events (20032 graft failures and 15869 deaths) over a 13-year period by using the data from the Scientific Registry of Transplant Recipients. Therefore, the 10-to-under-20 minute interval was chosen as the reference group. For each increment of 10 minutes of warm ischemia time was associated with an increased risk of death and graft failure. Interestingly, it was reported that after stratification by donor type (living vs. deceased donor) and delayed graft function status, the association between prolonged warm ischemia time and death/graft failure persisted. Longer times may be inevitable for some surgeries depending on the given donor and recipient factors even for the same surgeon. It is still not clear whether there is an ideal time below which there is no effect, however, strategies to reduce warm ischemia time like increasing surgical skills in simulation laboratories in order to achieve anastomosis times less than 30-35 minutes should be encouraged. In addition, better documentation of warm ischemia during surgery might help predict the future outcomes.

Yarkın Kamil Yakupoğlu, MD

\section{Re: Rac1-dependent Lamellipodial Motility in Prostate Cancer PC-3 Cells Revealed by Optogenetic Control of Rac1 Activity}

\author{
Kato T1, Kawai K2, Egami Y2, Kakehi Y1, Araki N2 \\ 1Kagawa University Faculty of Medicine, Department of Urology, Kagawa, Japan \\ 2Kagawa University Faculty of Medicine, Department of Histology and Cell Biology, Kagawa, Japan
}

PLoS One 2014;9:e97749. doi: 10.1371/journal.pone.0097749.

\section{EDITORIAL COMMENT}

Optogenetics is a biological method which includes the use of light to control cells in living tissue as neurons that have been genetically modified to express light sensitive ion channels. The key reagents used in optogenetics are light-sensitive proteins. Neuronal control is achieved using optogenetic actuators like channelrhodopsin, halorhodopsin and archaerhodopsin, while optical recording of neuronal activities can be made with the help of optogenetic sensors for calcium (GCaMP), vesicular release (synaptopHluorin), neurotransmitter (GluSnFRs) or membrane voltage (Arcclightining, ASAP1). In this study, the authors suggested Rac1-dependent lamellipodial motility in prostate cancer PC-3 cells revealed by optogenetic control of photoactivatable Rac1 activity. The lamellipodium plays an important role in invasion and metastasis of cancer cells for cell migration. Rac1 recognized as a main player in the formation of lamellipodium. They revealed the role of phosphatidylinositol 3-kinase (PI3K) in Rac1-dependent lamellipodial motility in PC-3 prostate cancer cells. Especially, inhibition of the neural hyperactivity in the micturation centers regulated by optogenetic application is a new therapeutic approach used to treat irreversible neurologic bladder dysfunction. In addition to the urooncology and neurourology, optogenetic researches will be focused on andrology (i.e. 5-hydroxy tryptamine 1A receptors). Optogenetic is a promising new field of research that will be applied in the treatment of various urological diseases in the future. 